\title{
Time series analysis of malaria in Afghanistan: using ARIMA models to predict future trends in incidence
}

\author{
Mohammad Y. Anwar ${ }^{1 *}$, Joseph A. Lewnard ${ }^{2}$, Sunil Parikh² and Virginia E. Pitzer ${ }^{2}$
}

\begin{abstract}
Background: Malaria remains endemic in Afghanistan. National control and prevention strategies would be greatly enhanced through a better ability to forecast future trends in disease incidence. It is, therefore, of interest to develop a predictive tool for malaria patterns based on the current passive and affordable surveillance system in this resourcelimited region.

Methods: This study employs data from Ministry of Public Health monthly reports from January 2005 to September 2015. Malaria incidence in Afghanistan was forecasted using autoregressive integrated moving average (ARIMA) models in order to build a predictive tool for malaria surveillance. Environmental and climate data were incorporated to assess whether they improve predictive power of models.

Results: Two models were identified, each appropriate for different time horizons. For near-term forecasts, malaria incidence can be predicted based on the number of cases in the four previous months and 12 months prior (Model 1); for longer-term prediction, malaria incidence can be predicted using the rates 1 and 12 months prior (Model 2). Next, climate and environmental variables were incorporated to assess whether the predictive power of proposed models could be improved. Enhanced vegetation index was found to have increased the predictive accuracy of longer-term forecasts.
\end{abstract}

Conclusion: Results indicate ARIMA models can be applied to forecast malaria patterns in Afghanistan, complementing current surveillance systems. The models provide a means to better understand malaria dynamics in a resourcelimited context with minimal data input, yielding forecasts that can be used for public health planning at the national level.

Keywords: Malaria, Prediction, Afghanistan, Environment, Autoregressive model

\section{Background}

Afghanistan is a landlocked country located at the crossroads of several geographical regions [1]. Although generally arid, there are numerous rain- and snowfed rivers [2], where historically human settlements formed at their surroundings, providing fertile ground for mosquito-borne diseases such as malaria. Major malaria vectors in the country are Anopheles stephensi, Anopheles superpictus, Anopheles hyrcanus, Anopheles

\footnotetext{
*Correspondence: manwar6@jhu.edu

${ }^{1}$ Department of International Health, Johns Hopkins Bloomberg School of Public Health, Baltimore, MD, USA

Full list of author information is available at the end of the article
}

pulcherrimus, Anopheles culicifacies, and Anopheles fluviatilis $[3,4]$. The major species are Plasmodium vivax (70-95\%), followed by Plasmodium falciparum [5, 6]. Malaria is endemic and seasonal in Afghanistan and the surrounding region $[7,8]$. Although varying figures are given for the number of people at risk for malaria $[5,9$, 10] the consensus is that significant numbers reside in malaria-endemic regions, notably in the semiarid eastern provinces, rice growing northern provinces, and greener areas under $1500 \mathrm{~m}$ in elevation [11]. In recent times, there have also been outbreaks of malaria in nontraditional highland provinces above $2000 \mathrm{~m}$, where malaria transmission was previously not believed to 
occur (e.g. Bamiyan province in the year 2000, with elevation of over $2400 \mathrm{~m}$ ) [12].

A particular problem with understanding the dynamics of malaria in Afghanistan is the scarcity of consistent and systemic information sources due to a combination of lack of infrastructure and constant civil unrest. In this unstable setting, not much is known about the intensity, magnitude, and temporal dependence of epidemic patterns over time. Only recently has a systemic surveillance system been put in place [13], but the scope is limited and mostly confined to accessible regions. Reporting is based on passive case finding from facilities by health professionals. It is retrospective and often late to detect emerging patterns. Hence, a tool to actively predict future trends is needed, especially one with the capability of producing good results in a resource-poor and wartorn setting like Afghanistan.

The increasing availability of data on climatic, geographic, and environmental determinants of transmission encourages consideration of these factors together with clinical data to prepare early warning signals of changing malaria trends in modern public health surveillance [6]. It has been proposed that variables like air temperature [14], rainfall [15], altitude [16], humidity [17], vegetation index [18], and even surface water fraction [19] increase predictive power of malaria models [20], not only for short periods, but also over longer timescales [21]. Tools used to measure the association between these factors and malaria patterns have included linear regression [22], Poisson regression [23], Spearman's correlation [24], non-linear methods [25], and autoregressive time series methods [26].

In this paper, an autoregressive integrated moving average (ARIMA) model was used, applied to time series data of malaria incidence in Afghanistan. The model looks for temporal dependence between successive observations [27]. Due to the transmissibility and seasonality of malaria, models with an ARIMA structure have more predictive power compared to other methods [28]; such models have been applied to predict numerous infectious diseases with similar periodic patterns over the past decades [29, 30]. Another advantage of the ARIMA approach is the relative simplicity and stability of the model in predicting malaria cases in a context where political unrest and poor resources lead to a lack of detailed data, which makes it difficult to calculate parameters needed for construction of more complex models of malaria [31]. Remotely-sensed climate and environmental data were incorporated to test associations with climate and improve the predictive power of proposed model [32].

\section{Methods}

\section{Malaria data}

Models forecasting monthly malaria incidence throughout Afghanistan were developed. Data were available from cases reported nationwide across all regions of Afghanistan over the period from January 2005 to September 2015 through Health Management Information System (HMIS), a Ministry of Public Health-operated database [33], which collects reports from public health facilities accessed by over $85 \%$ of the population [34]. These reports capture passively detected cases from the public health system, and include both parasitologically confirmed and clinically suspected cases referred to outpatient departments. Inclusion of clinically suspected cases as numerator makes results prone to overestimation, but after accounting for significant underreporting of confirmed cases due to the lack of laboratory facilities, and the fact that around $15 \%$ of the population still lack access to health services and could have higher incidences compared to those under coverage, the numbers approximate those reported by the World Health Organization (WHO) for Afghanistan (the only available reference) [5].

No public census has been conducted in Afghanistan since 1979 [35], and other sources of demographic data [e.g. WHO, International Monetary Fund (IMF), Central Statistics Office (CSO)] cannot be corroborated with each other. In addition, utilization of health services was not homogenous throughout the study period (Fig. 2c), as the number facilities has risen from under 1000 to over 2000 centres since 2004 . Hence, data on the total monthly new outpatient department visits were used as denominator in order to control for demographic and reporting trends. To verify that this did not lead to a bias in the trends over time due to recent changes in outpatient health service utilization occurring primarily in regions of either low or high malaria incidence, the overall of trend of malaria obtained after adjustment was compared with the weighted average of individual trends of provinces adjusted for their level of health service utilization.

\section{Climate/environmental data}

Satellite-based measures of meteorological and environmental variables used to aid forecasting were available from the earth observing system data and information system (EOSDIS). Precipitation ( $\mathrm{mm} / \mathrm{month}$ ), surface relative humidity (daily data, averaged by month), enhanced vegetation index (EVI) [36] (monthly average land greenness fraction), and surface air temperature (daily data, averaged by month) were assessed for Afghanistan as potential predictors. Both Malaria and climate data were provided as Additional files 1, 2, 3, 4 and 5. 


\section{Statistical procedure}

ARIMA models were developed to forecast malaria incidence based on temporal autocorrelation present in the incidence data. The dataset was split into a training period (January 2005 to December 2013), used as a platform for creating the ARIMA models, and a validation period (January 2014 to September 2015), which was used to test the models' predictive ability.

ARIMA models provide $n$-step-ahead predictions based on patterns of temporal dependence in time series data. The notation $(p, d, q) \times(P, D, Q)_{S}$ describes the composition of temporal patterns considered for forecasting: these include autocorrelation over a maximum of $p$ months or over $P$ periods, each of length $S=12$ months in our dataset; differencing over $d$ adjacent months or $D$ periods; and moving averages sustained over $q$ months or $Q$ periods. To determine patterns best describing the malaria time series, we followed the Box-Jenkins approach to ARIMA model selection, consisting of three steps [37]. First, malaria incidence was plotted against time to detect and correct for non-stationarity of the time series (Fig. 2), and identified autoregressive and moving average terms needed by calculating the autocorrelation (ACF) and partial autocorrelation (PACF) functions. Next, models of varying orders were fitted, and compared via the Akaike information criterion (AIC) [38] to assess improvements in fit while penalizing model complexity. Last, temporal autocorrelation was confirmed to have been no longer present in model residuals using the Ljung-Box test [39].

The selected models were used to generate forecasts for the validation period from January 2014 to September 2015 as 1-, 2-, 3-, 6-, and 12-month ahead forecasts. The rationale was to find which model works better for realtime, short-term surveillance objectives as compared to longer-term (up to yearly) prediction of future malaria patterns.

Out-of-sample forecast accuracy across models was compared by calculating the mean square error (MSE) and the predictive $R^{2}$, which is equal to 1 - (mean squared error)/(variance of the time series). Similar to the coefficient of determination, predictive $R^{2}$ tends toward one as models explain more observed heterogeneity in a time series, but can also take on values less than zero when the mean of the time series would provide a better estimate than model-based forecasts. Lastly, model forecasts, along with 95\% prediction intervals, were plotted and compared against the observed data between January 2014 and September 2015.

It was evaluated whether incorporating meteorological and environmental variables improved the models' fit and forecasting ability. Predictors were selected using a standard "pre-whitening" approach to identify whether each variable and the malaria time series were associated after adjusting for shared patterns of temporal dependence [40]. ARIMA models were selected and fitted to each climatic predictor, then fitted ARIMA models of the same order to the malaria time series. The cross-correlation function was evaluated between residuals series from the two models to identify lags at which anomalies in the climate variables explained unaccounted-for heterogeneity in malaria incidence. Lags found to be significantly correlated with malaria residuals were incorporated into the base ARIMA model as external regressors. Models with external regressors were used for both short- and long-term predictions; regressors were forecasted with the corresponding number of time steps before being incorporated into the malaria prediction models whenever predictive horizons exceeded the available data on these variables.

$\mathrm{R}$ statistical package ( $\mathrm{R}$ Core Development Team, Vienna) and Stata v12 (StataCorp, College Station, TX) were used to carry out the analyses.

\section{Results}

The dataset covers 129 months, starting from January 2005 to September 2015. The total number of suspected (including confirmed) malaria cases reported throughout the period was $2,243,452$ with a mean of 20,772 clinical cases per month, and standard error of 1097 cases. The number of reported cases per month ranged from 4309 to 47,779 , consistent with the seasonal nature of malaria in the country. Indeed, looking at the seasonal distribution of cases over the years (Fig. 1a), malaria cases peak between June-September, around the time when temperature is high and rainfall low (Fig. 1b, d), and lag vegetation variation by few months (Fig. 1c). Geographically, in descending order, eastern $(1,351,530)$, north eastern $(366,635)$, northern $(239,230)$, southern $(145,220)$, central $(87,227)$, and western $(53,610)$ regions report the most cases.

Malaria notifications have proportionally declined relative to the total number of outpatient visits consistently since the beginning of 2005, with seasonal pattern of 12-month in length, which has decreased in amplitude over time (Fig. 2a). The overall (linear) trend in malaria cases per 1000 outpatient visits was -27 (CI -34, -21) per year, compared with a population-weighted mean of $-32(\mathrm{CI}-47,-18)$ cases per 1000 outpatient visits per year for provinces individually; thus the rate of decline was statistically the same for provinces as for the country as whole.

The time series data were log-transformed then differenced to stabilize the variance and remove the linear trend, respectively (Fig. 3a). The resulting time series exhibits a faint, statistically non-significant second 

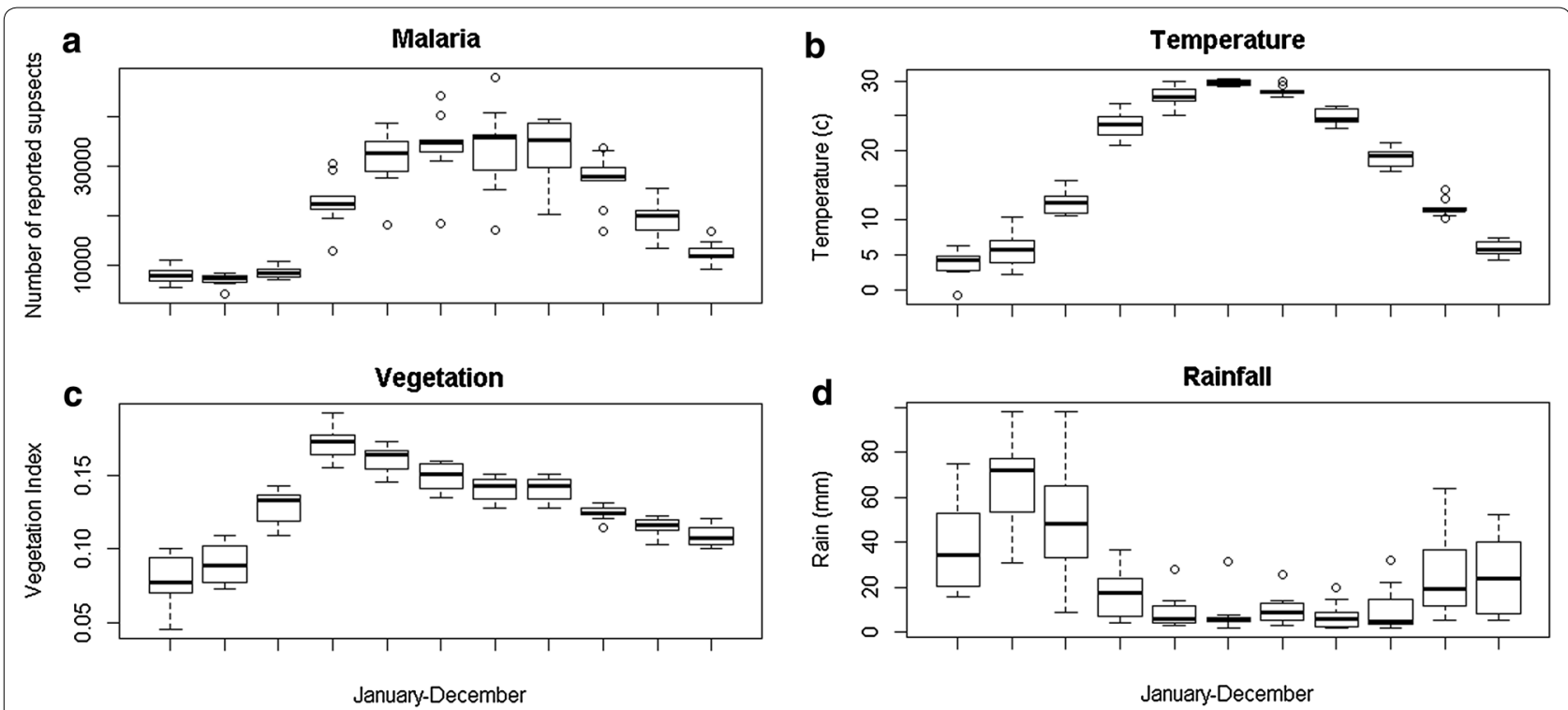

Fig. 1 Seasonal variation of malaria and environmental variables (2005-2014). From top left in clock wise order: a monthly variation of malaria, b monthly variation of Temperature, $\mathbf{d}$ monthly variation of rainfall, $\mathbf{c}$ monthly variation of vegetation index

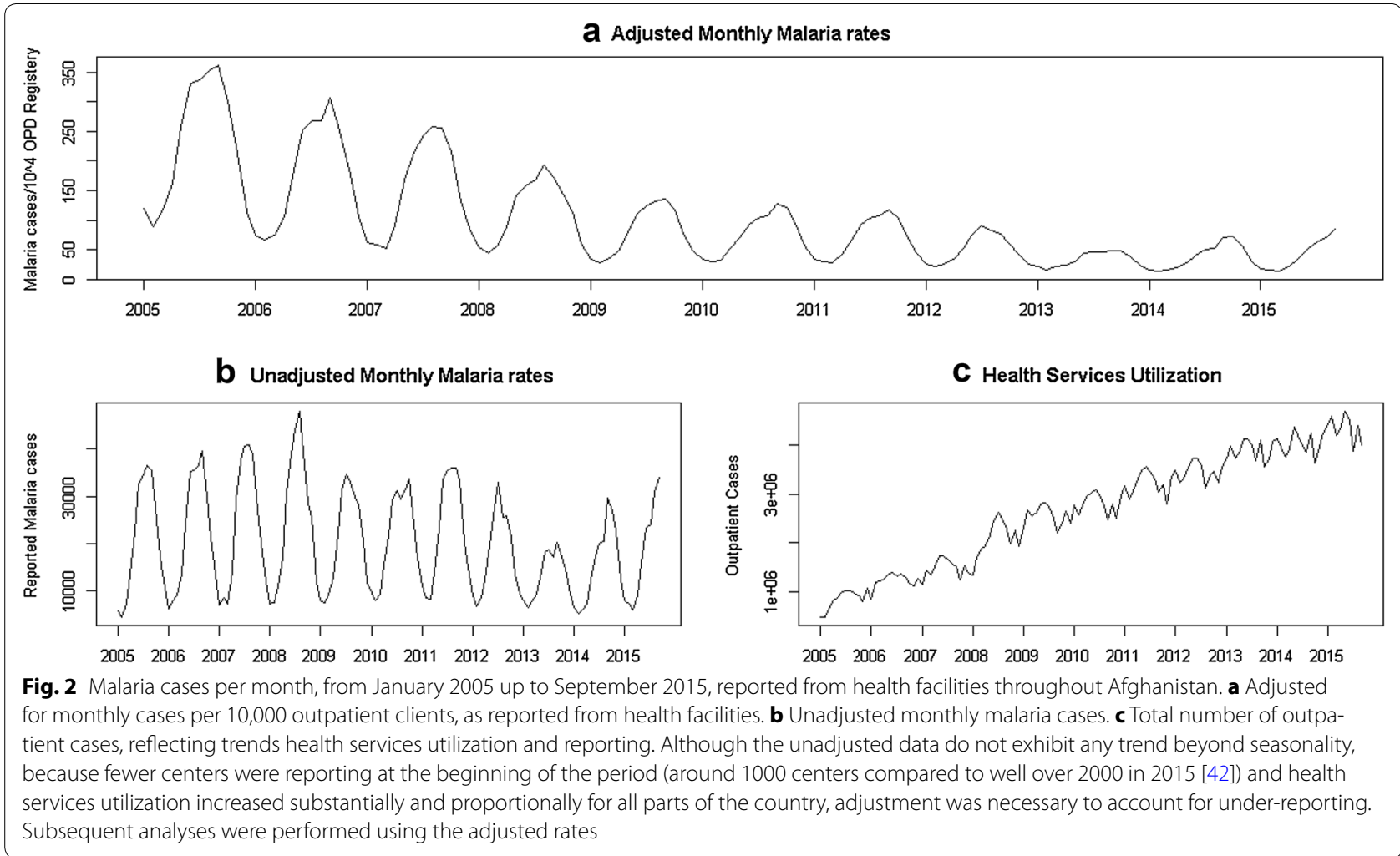

periodic peak after the first, possibly due to distinct $P$. vivax and P. falciparum cycles [41]. Based on the ACF and PACF patterns (Fig. 3b, c), an ARIMA model of order $(4,1,1) \times(1,0,1)_{12}, \quad($ Model 1, AIC $=-145.02)$ was selected and fitted (with the consideration of first degree differencing). The residuals did not show a statistically significant autocorrelation pattern (Ljung-Box test $p=0.4067$ ) (Additional file 6: Annex 1; Table 1). 


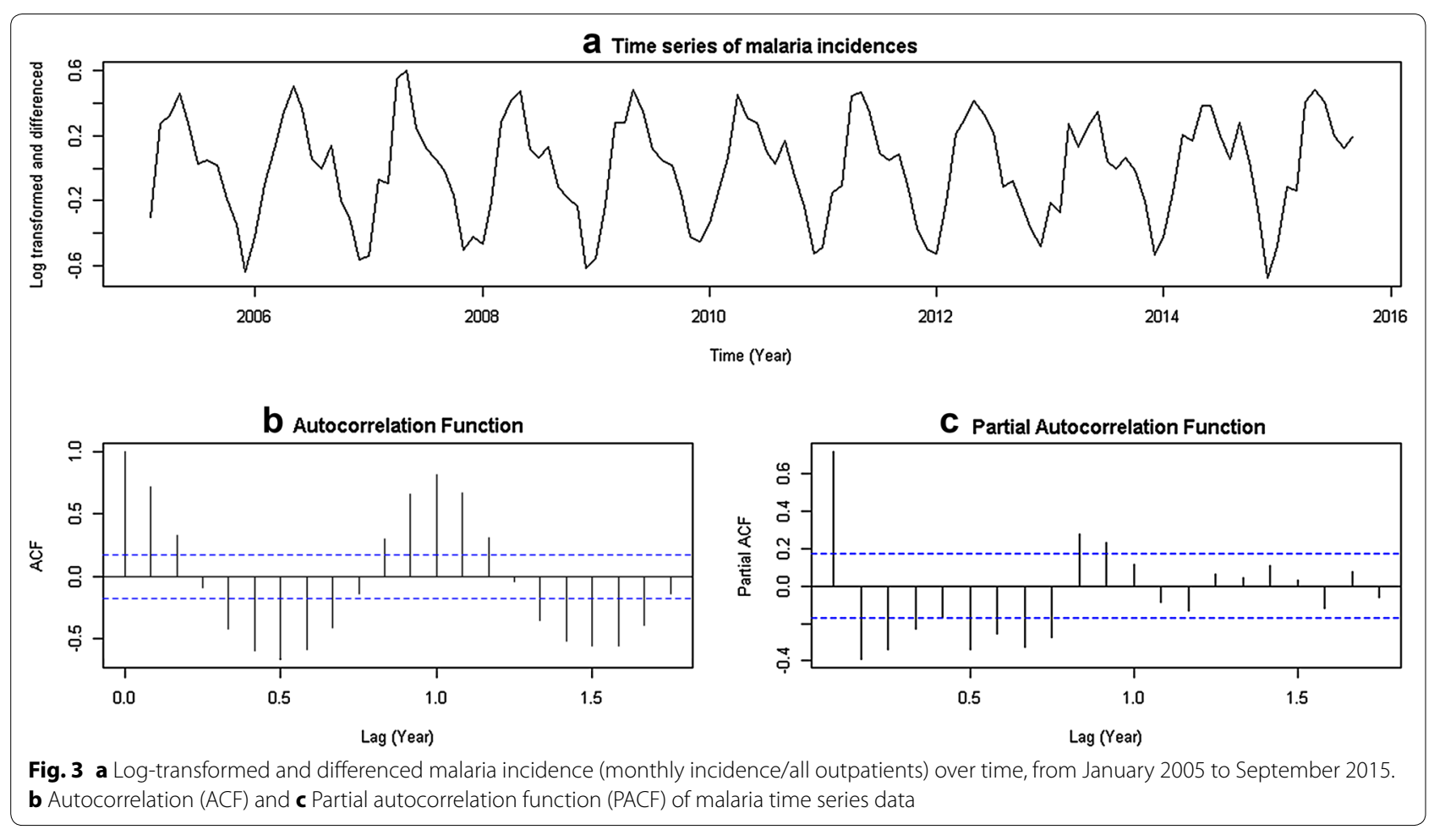

For comparison, a more parsimonious ARIMA model of order $(1,1,1) \times(1,0,1)_{12}($ Model 2 , AIC $=-132.18)$ was also considered; however, a marginal degree of temporal autocorrelation persisted in the residuals of Model 2 $(p=0.052)$ (Additional file 6: Annex 1).

Both models were used to compare the observed versus predicted malaria incidence from January 2014 to September 2015. For one-step ahead predictions, the estimated values show less dispersion using Model 1 compared to Model 2 (reduction in MSE of 10\%) (Table 2); this suggests Model 1 may be better suited for short-term, out-of-sample malaria forecasting. For longer-term prediction, the MSE and predictive $R^{2}$ of both models were compared. The values estimated for 2- , 3- , 6- and 12-step ahead approaches exhibit generally better predictive power for Model 2 at longer time steps, despite its poorer within-sample fit as measured by AIC (Table 3 ).

Subsequently it was assessed whether incorporating external climate regressors improved the predictive power of proposed models. The correlation coefficients between the covariate data and the residuals of the ARIMA model fit to the time series over a range of lags are presented in Additional file 7: Annex 2. Using the prewhitening approach, it was found that only EVI with a lag of 2 months was significantly correlated with the malaria outcome (pairwise correlation $=0.2012, p=0.0318$ ) (Additional file 7: Annex 2). After fitting Models 1 and 2 with EVI as an external regressor, we found the simpler model (Model 2) demonstrated improved within-sample model fit (AIC $=-147.69$ ), whereas fit for Model 1 was not improved (AIC $=-121.99$ ) (Table 2). Incorporating EVI marginally improved the accuracy of one-month ahead forecasts from Model 2 (Table 2). Even though the forecasted vegetation index itself was not a significant predictor, adjusting for EVI in Model 2 affected the estimates of the other contributing parameters, in particular strengthening the non-seasonal autoregressive and moving average terms (Table 1), leading to a better overall model fit. As found in the earlier analysis, Model 2 had generally better longer-term predictive power compared to Model 1, and accounting for lag-2 EVI further improved the predictive power by a small factor (Table 3 ).

Figure 4 demonstrates the 2-, 3-, 6-, and 12-step ahead predictions and fitted values for the multiplicative ARIMA $(4,1,1) \times(1,0,1)_{12}$ model (Model 1), $(1,1,1)$ $\times(1,0,1)_{12}$ model (Model 2), and Model 2 with lag-2 EVI. Model forecasts for the expected number of clinically suspected malaria cases up to December 2016 are presented in Additional file 8: Annex 3, using 12-step ahead predictions from Model 2; these estimates depend on the assumptions highlighted in Additional file 8: Annex 3.

\section{Discussion}

While the overall number of malaria cases reported to the Health Management Information System in Afghanistan has remained fairly constant, analysis indicates 
Table 1 Coefficients and standard errors of parameters of both ARIMA models

\begin{tabular}{lcc}
\hline & Coefficient & Standard error \\
\hline Coefficients, and standard errors of the parameters of the ARIMA Model \\
$1\left[(4,1,1) \times(1,0,1)_{12}\right]$ & & \\
Intercept & -0.0197 & 0.0186 \\
Non-seasonal AR(1) & 0.6745 & 0.1342 \\
Non-seasonal AR(2) & 0.0120 & 0.1162 \\
Non-seasonal AR(3) & -0.0068 & 0.1161 \\
Non-seasonal AR(4) & -0.3169 & 0.0991 \\
Non-seasonal MA(1) & -0.7784 & 0.0890 \\
Seasonal AR(1) & 0.9998 & 0.0016 \\
Seasonal MA(1) & -0.9576 & 0.1424 \\
Coefficients, and standard errors of the parameters of the ARIMA Model \\
$2\left[(1,1,1) \times(1,0,1)_{12}\right]$ & & \\
Intercept & -0.0209 & 0.0840 \\
Non-seasonal AR(1) & -0.0035 & 0.0028 \\
Non-seasonal MA(1) & -0.0524 & 0.0163 \\
Seasonal AR(1) & 0.9996 & 0.0000 \\
Seasonal MA(1) & -0.9154 & 0.0094 \\
Coefficients, and standard errors of the parameters of the ARIMA Model \\
$2\left[(1,1,1) \times(1,0,1)_{12}\right]$ Lag2 EVI & \\
Intercept & -0.0408 & 0.0953 \\
Non-seasonal AR(1) & 0.7777 & 0.0729 \\
Non-seasonal MA(1) & -1.0000 & 0.0033 \\
Seasonal AR(1) & 0.9993 & 0.0023 \\
Seasonal MA(1) & -0.8782 & \\
\hline
\end{tabular}

Table 2 Comparison of 1-step ahead models with and without external regressors

\begin{tabular}{lllll}
\hline ARIMA model & $\begin{array}{l}\text { Lag-2 EVI coef- } \\
\text { ficient }\end{array}$ & Standard error & AIC & MSE \\
\hline $\begin{array}{l}\text { Model 1 } \\
\text { Model 1 with }\end{array}$ & 0.6287 & 0.4719 & -145.02 & 0.0088 \\
$\quad$ Lag-2 EVI & & & -121.99 & 0.0142 \\
$\begin{array}{l}\text { Model 2 } \\
\text { Model 2 with } \\
\text { Lag-2 EVI }\end{array}$ & 0.1728 & 0.7304 & -132.18 & 0.0098 \\
\hline
\end{tabular}

malaria incidence and the intensity of seasonal epidemics as a proportion of the total number of outpatient clients have been steadily declining (by greater than $75 \%$ ) since 2005 [5]. This perhaps can be attributed to recent efforts to expand health services in the country [34], which may have resulted in a general drop in communicable diseases, including malaria [43]. Furthermore, wider implementation of preventive measures such as insecticide-treated nets in recent years, even in remote and impoverished regions [44], have been shown to have a negative correlation with malaria incidence [45]. In addition, substantial increase in number of trained health worker in recent years helped maximize the effect of malaria control programmes [46]. It might be even possible to credit these designed intervention as the major determinant of malaria trend in the country.

After adjusting for these trends in malaria incidence, two ARIMA models were evaluated. The best fit to the data was obtained with a $(4,1,1) \times(1,0,1)_{12}$ model. Thus, the number of malaria cases in a given month can be estimated based on the number of cases occurring 1, 2, 3, 4, and twelve months before, after adjustment for negative seasonal and non-seasonal moving averages (i.e. a slight decrease in average cases in a given month compared to the prior month and the same month but in the previous year, respectively). Although this model is a good fit for short-term 1-step ahead prediction, it does not perform as well for longer-term predictions.

The second model, which is a $(1,1,1) \times(1,0,1)_{12}$ model, indicates that the number of malaria cases can be estimated from cases occurring one month and 12 months before. Again, the moving average parameters indicate a drop in magnitude of average cases in a given month compared to 1 and 12 months before. Although this model does not provide as good a fit to the observed data as the model above, it nonetheless has better longterm predictive power, and estimated averages remain close to the observed data. Furthermore, the fit and predictive power of the second model can be improved with the addition of environmental variables.

Several climate and environmental variables have been associated with malaria incidence $[14,20]$. To measure associations between these variables and malaria incidence in Afghanistan, the data were prewhitened to facilitate the evaluation of possible correlation between two time series after accounting for temporal and seasonal autocorrelation. In the absence of pre-whitening, significant correlations existed between malaria and average monthly rainfall ( $0-3$ month lags), vegetation ( $0-3$ month lags), and temperature (0-3 month lags) (Additional file 7: Annex 2 ), which are likely attributable to common seasonal patterns. After pre-whitening, it was found that only EVI had a significant association with malaria at a lag of 2 months. Thus, average malaria cases might depend on how green the environment was (i.e. the amount of vegetation covering the environment, as measured by EVI) 2 months before.

Incorporating EVI as an external regressor at a lag of 2 months improved the predictive power of Model 2 , especially for 2-, 6- and 12-steps ahead predictions; the same did not happen with Model 1. Although the improvement is not substantial, it is nonetheless helpful 
Table 3 Model forecasting and validation for 2-, 3-, 6-, and 12-step ahead predictions for both models, with or without the external regressor (EVI at a lag of 2 months) over the period from January 2014 to September 2015

\begin{tabular}{|c|c|c|c|c|c|c|c|c|}
\hline \multirow[t]{4}{*}{ Steps ahead } & \multicolumn{8}{|c|}{ Model } \\
\hline & \multicolumn{4}{|c|}{ Model 1} & \multicolumn{4}{|c|}{ Model 2} \\
\hline & \multicolumn{2}{|c|}{$(4,1,1) \times(1,0,1) 12$} & \multicolumn{2}{|c|}{$\begin{array}{l}(4,1,1) \times(1,0,1) 12-\operatorname{lag} 2 \\
\text { vegetation }\end{array}$} & \multicolumn{2}{|c|}{$(1,1,1) \times(1,0,1) 12$} & \multicolumn{2}{|c|}{$\begin{array}{l}(1,1,1) \times(1,0,1) 12- \\
\text { lag } 2 \text { vegetation }\end{array}$} \\
\hline & MSE & R2 & MSE & R2 & MSE & R2 & MSE & R2 \\
\hline 2 & 0.041 & 0.897 & 0.045 & 0.887 & 0.045 & 0.887 & 0.041 & 0.897 \\
\hline 3 & 0.075 & 0.812 & 0.075 & 0.810 & 0.064 & 0.838 & 0.066 & 0.833 \\
\hline 6 & 0.113 & 0.634 & 0.132 & 0.666 & 0.107 & 0.729 & 0.107 & 0.729 \\
\hline 12 & 0.160 & 0.595 & 0.168 & 0.575 & 0.145 & 0.633 & 0.144 & 0.638 \\
\hline
\end{tabular}

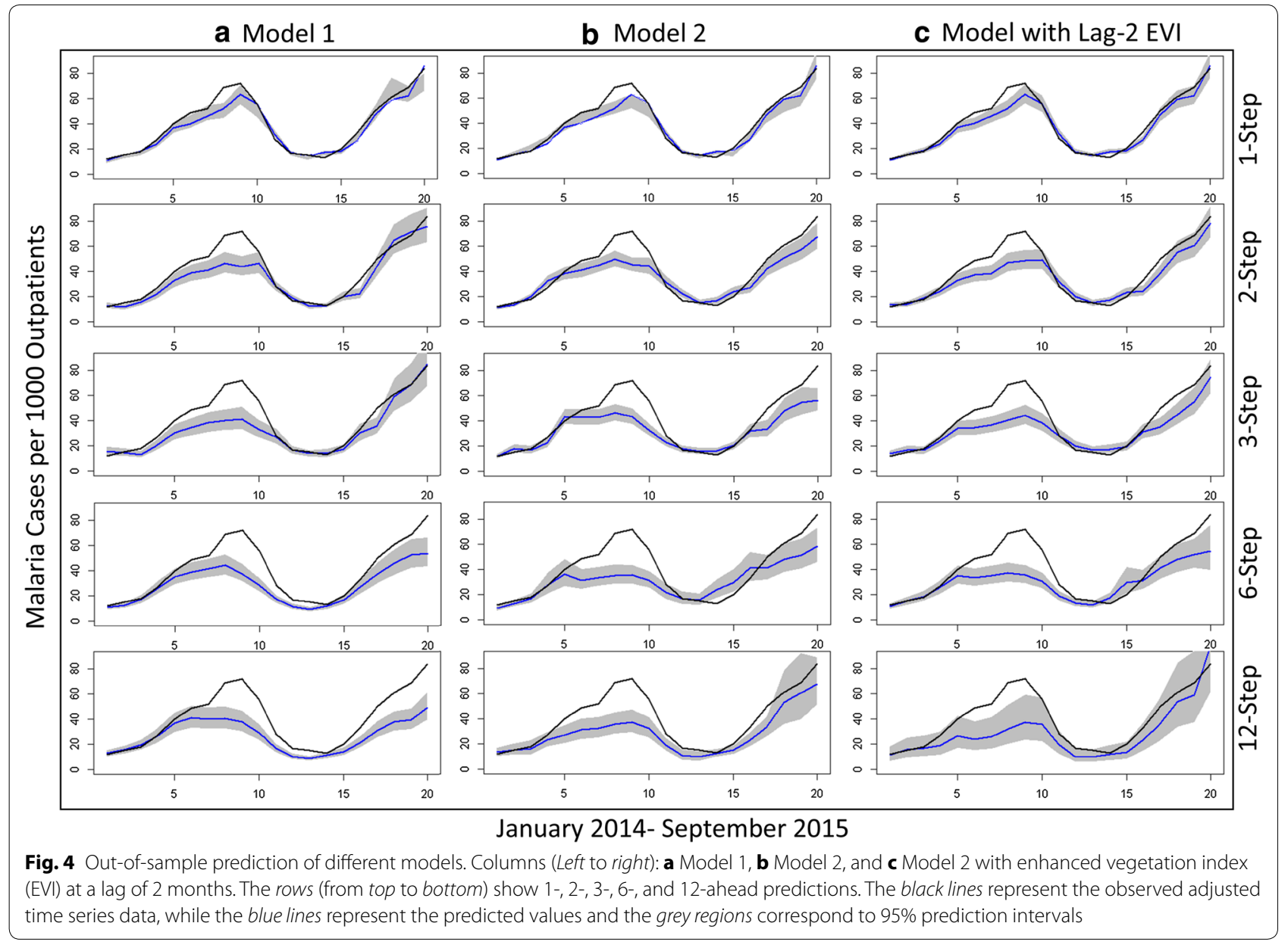

to empower surveillance bodies in the country to sharpen their predictions, and to understand how much of a role environment plays in malaria dynamics in the country.
The finding that vegetation is correlated with malaria cases in Afghanistan is in line with other studies using remote sensing data in close or distant regions that found 
such association with lags between (0-3) months [18, 47]. Although strong evidence exists for an effect of temperature and rainfall on malaria, results did not point to any statistically significant correlations with these variables after controlling for the seasonal and autoregressive patterns. The reason might be our assumption that average monthly temperature and rainfall were the same across the entire country, although Afghanistan is geographically diverse [48]. Change in temperature does not necessarily equate to a rise in malaria in some parts of the country, particularly in regions which experience high temperatures on average; in fact, higher temperature $\left(>31 \mathrm{C}^{0}\right)$ can have an inhibitory effect on the mosquito life cycle [49]. Thus, the negative correlation of temperature in some corners of the country is perhaps balanced by a positive correlation in others. Thus, vegetation seems to be a better predictor of malaria at the country level, because greenness is not only an indicator for bountifulness of environments for growth of mosquitos, but also moisture and appropriate temperature, both of which are relevant to malaria. A study of malaria patterns in different Afghan provinces, using local scale data from 2004 to 2007 , also pointed to vegetation as the strongest predictor of malaria [50], as well as another geospatial study of vivax malaria, the dominant type in the country in 2005 [9].

Declines in malaria incidence in Afghanistan and elsewhere have prompted a paradigm shift from the national level action to region-limited interventions, especially in malaria hotspots. Indeed, since the early 2000s, Afghanistan has steadily come closer to realizing such a scenario. However, these efforts have recently been hampered for two reasons: (1) The required funds to initiate the next phase of the malaria control strategy have yet to be realized, despite efforts to shift the strategy to more local control efforts since 2012 (personal communication with an official in the Ministry of Public Health). (2) The recent deterioration of security (particularly since 2014) throughout the country has raised concerns about potential increases in malaria incidence [51]. The government's lack of effective territorial control over many malaria burdened areas make it untenable to move toward region-focused initiatives. In light of Afghanistan's current context, it is tenable that a national-level predictive tool is still very much required, particularly one that can be cost-efficient, to at least ensure the success in the first phase of malaria control in this resource-poor setting.

Most malaria studies in Afghanistan have either focused upon general trends of infection in recent years [45], or the implementation of preventive measures and their effects on the burden of malaria [44]. In general, studies which have assessed the correlation of environmental variables and malaria incidence have tended to be focused on smaller geographic scales [52, 53]. Analysis conducted in this paper complements these efforts by attempting to build a predictive tool that can be used to forecast malaria cases at a national level based on observations from a passive surveillance system that is currently in place. In a country such as Afghanistan, where infrastructure is limited, a system that can accurately predict future malaria trends would be a great asset for public health planning and resource allocation. In addition, proposed model forecasts malaria incidence based solely on passive surveillance data and widely available climate indices, enabling short-term predictions that may provide useful indicators of lapses in malaria control in a setting of ongoing civil unrest. Not only were proposed models able to forecast malaria up to one year ahead with minimum data inputs, but they also provide a means to better understand malaria dynamics in a setting disproportionately affected by lack of resources, ongoing civil unrests, and climate change [54].

\section{Additional files}

Additional file 1. Malaria Metadata.

Additional file 2. Area-Averaged of CMG 0.05 Deg Monthly EVI monthly 0.05 ().

Additional file 3. Area-Averaged of Air temperature at surface (Daytime/ Ascending) ().

Additional file 4. Area-Averaged of Precipitation Rate monthly 0.25 ().

Additional file 5. Area-Averaged of Relative Humidity at Surface (Daytime/Ascending) ().

Additional file 6: Annex 1. Right side: Autocorrelation (ACF) and partial autocorrelation (PACF) functions of the residuals from ARIMA model (1, $0,1) \times(1,0,1)_{12}$ on log-transformed, differenced data. Left side: ACF and PACF of the residuals from ARIMA model $(4,0,1) \times(1,0,1)_{12}$ on logtransformed, differenced data.

Additional file 7: Annex 2. Pairwise correlation between malaria ARIMA model residuals and external regressor residuals at different lags, after pre-whitening (removing trends and seasonality and fitting ARIMA models to each) (first table). In preliminary analyses, statistically significant correlation was observed between rain and humidity $(r=0.7032, p<$ $0.001)$; subsequently, humidity was dropped after it was found not to add meaningful information. Had we not performed pre-whitening, statistically significant correlations existed between malaria and other variables at every lag we analyzed.

Additional file 8: Annex 3. Approximate estimation of malaria suspects expected up to December 2016, based on Model 2 with 2-Lag Vegetation. This estimate may be taken with following considerations: 1- Assuming linear trend of malaria stays the same as the Model predict. 2- Incidences not reported to the system remain small or negligible. The numbers calculated are incidence rate per 10000 of service users in the country

\section{Authors' contributions}

MYA obtained data, wrote the draft, coded and carried out the statistical analysis. JL wrote statistical codes, contributed to data analysis, and provided feedback on the manuscript. SP reviewed the contents, suggested technical insights, and helped revise the manuscript. VEP supervised the study, contributed to data analysis, revised the manuscript, and finalized the draft. All authors read and approved the final manuscript. 


\section{Author details}

${ }^{1}$ Department of International Health, Johns Hopkins Bloomberg School of Public Health, Baltimore, MD, USA. ${ }^{2}$ Department of Epidemiology of Microbial Diseases, Yale School of Public Health, New Haven, CT, USA.

\section{Acknowledgements}

We sincerely thank the Afghan Ministry of Public Health, and in particular Dr. Sayed Yaqoob Azimi, the Head of Health Management Information System department, for providing data that was used for our analysis.

\section{Availability of data and materials}

The dataset supporting the conclusions of this article is provided as additional file to the journal.

\section{Competing interests}

The authors declare that they have no competing interests.

\section{Received: 28 July 2016 Accepted: 4 November 2016}

Published online: 22 November 2016

\section{References}

1. Wilber D. Afghanistan: its people, its society, its culture. New Haven: HRAF Press; 1962

2. Brookfield M. The evolution of the great river systems of southern Asia during the Cenozoic India-Asia collision: rivers draining southwards. Geomorphology. 1998;22:285-312.

3. Rowland M, Mohammed N, Rehman H, Hewitt S, Mendis C, Ahmad M, et al. Anopheline vectors and malaria transmission in eastern Afghanistan. Trans R Soc Trop Med Hyg. 2002;96:620-6.

4. Youssef R, Safi N, Hemeed H, Sediqi W, Naser JA, Butt W. National malaria indicators assessment. Afghan Annu Malaria J. 2008;2008:37-49.

5. WHO. World malaria report summary. Geneva: World Health Organization; 2015. p. 2015

6. Edlund S, Davis M, Douglas J, Kershenbaum A, Waraporn N, Lessler J, et al. A global model of malaria climate sensitivity: comparing malaria response to historic climate data based on simulation and officially reported malaria incidence. Malar J. 2012;11:331.

7. Lindberg K. Malaria in Afghanistan. Riv Malariol. 1949;28:1-54

8. Cutler JC. Survey of venereal diseases in Afghanistan. Bull World Health Organ. 1950;2:689.

9. Brooker S, Leslie T, Kolaczinski K, Mohsen E, Mehboob N, Saleheen S, et al. Spatial epidemiology of Plasmodium vivax Afghanistan. Emerg Infect Dis. 2006;12:1600-2

10. Zakeri S, Safi N, Afsharpad M, Butt W, Ghasemi F, Mehrizi A, et al. Genetic structure of Plasmodium vivax isolates from two malaria endemic areas in Afghanistan. Acta Trop. 2010;113:12-9.

11. Faulde M, Hoffmann R, Fazilat $K$, Hoerauf A. Malaria reemergence in Northern Afghanistan. Emerg Infect Dis. 2007;13:1402-4.

12. Abdur Rab M, Freeman TW, Rahim S, Durrani N, Simon-Taha A, Rowland M. High altitude epidemic malaria in Bamian province, central Afghanistan. East Mediterr Health J. 2003;9:232-9.

13. Jawad M, Jamil A. Evaluation of measles surveillance systems in Afghanistan-2010. J Public Health Epidemiol. 2014;6:407.

14. Garske T, Ferguson N, Ghani A. Estimating air temperature and its influence on malaria transmission across Africa. PLoS ONE. 2013;8:e56487.

15. Thomson MC, Mason SJ, Phindela T, Connor SJ. Use of rainfall and sea surface temperature monitoring for malaria early warning in Botswana. Am J Trop Med Hyg. 2005;73:214-21.

16. Siraj A, Santos-Vega M, Bouma M, Yadeta D, Carrascal D, Pascual M. Altitudinal changes in malaria incidence in highlands of Ethiopia and Colombia. Science. 2014;343:1154-8.

17. Lyons CL, Coetzee M, Terblanche JS, Chown SL. Desiccation tolerance as a function of age, sex, humidity and temperature in adults of the African malaria vectors Anopheles arabiensis Patton and Anopheles funestus Giles. J Exp Biol. 2014;217:323-33.

18. Ricotta E, Frese S, Choobwe C, Louis T, Shiff C. Evaluating local vegetation cover as a risk factor for malaria transmission: a new analytical approach using ImageJ. Malar J. 2014;13:94.
19. Hirt C, Chen B, Jensen K, McDonald KC. Development of an early warning system for extreme rainfall, surface inundation, and malaria in East Africa. AGU Fall Meet Abstr. 2013;1:0066.

20. Thomson M, Doblas-Reyes F, Mason S, Hagedorn R, Connor S, Phindela $T$, et al. Malaria early warnings based on seasonal climate forecasts from multi-model ensembles. Nature. 2006;439:576-9.

21. Rogers DJ, Randolph SE. The global spread of malaria in a future, warmer world. Science. 2000;289:1763-6.

22. Craig MH, Kleinschmidt I, Nawn JB, Le Sueur D, Sharp BL. Exploring 30 years of malaria case data in KwaZulu-Natal, South Africa: part I. The impact of climatic factors. Trop Med Int Health. 2004:9:1247-57.

23. Teklehaimanot H, Lipsitch M, Teklehaimanot A, Schwartz J. Weatherbased prediction of Plasmodium falciparum malaria in epidemic-prone regions of Ethiopia I. Patterns of lagged weather effects reflect biological mechanisms. Malar J. 2004;3:41.

24. Bi P, Tong S, Donald K, Parton KA, Ni J. Climatic variables and transmission of malaria: a 12-year data analysis in Shuchen County China. Public Health Rep. 2003:118:65.

25. Zhou G, Minakawa N, Githeko A, Yan G. Association between climate variability and malaria epidemics in the East African highlands. Proc Natl Acad Sci USA. 2004;101:2375-80.

26. Wangdi K, Singhasivanon P, Silawan T, Lawpoolsri S, White N, Kaewkungwal J. Development of temporal modelling for forecasting and prediction of malaria infections using time-series and ARIMAX analyses: a case study in endemic districts of Bhutan. Malar J. 2010;9:251.

27. Helfenstein Ulrich. The use of transfer function models, intervention analysis and related time series methods in epidemiology. Int J Epidemiol. 1991;20:808-15

28. Nobre F, Monteiro A, Telles P, Williamson G. Dynamic linear model and SARIMA: a comparison of their forecasting performance in epidemiology. Statist Med. 2001:20:3051-69.

29. Ture M, Kurt I. Comparison of four different time series methods to forecast hepatitis A virus infection. Expert Syst Appl. 2006:31:41-6.

30. Luz PM, Mendes BV, Codeço CT, Struchiner CJ, Galvani AP. Time series analysis of dengue incidence in Rio de Janeiro. Brazil. Am J Trop Med Hyg. 2008;79:933-9.

31. Pascual M, Cazelles B, Bouma M, Chaves L, Koelle K. Shifting patterns: malaria dynamics and rainfall variability in an African highland. Proc Biol Sci. 2008;275:123-32.

32. Beck LR, Lobitz BM, Wood BL. Remote sensing and human health: new sensors and new opportunities. Emerg Infect Dis. 2000;63:217.

33. Chaudhery D, Gupta P, Kaushik S. Strengthening Government Health Management Information System (HMIS) and Innovative Monitoring Approaches in Micronutrient Demonstration Programs: experience from Three Asian Countries. EJNFS. 2015;5:896-7.

34. Acerra J, Iskyan K, Qureshi Z, Sharma R. Rebuilding the health care system in Afghanistan: an overview of primary care and emergency services. Int $J$ Emerg Med. 2009;2:77-82.

35. Khalidi N. Demographic Profile Of Afghanistan. Canberra, ACT, Australia International Population Dynamics Program, Dept. of Demography, Research School of Social Sciences, the Australian National University; 1989

36. Matsushita B, Yang W, Chen J, Onda Y, Qiu G. Sensitivity of the enhanced vegetation index (EVI) and normalized difference vegetation index (NDVI) to topographic effects: a case study in high-density cypress forest. Sensors. 2007;7:2636-51

37. Box G. Box and Jenkins time series analysis, forecasting and control A very british affair. London: Palgrave Macmillan UK; 2013. p. 161-215.

38. Bozdogan $\mathrm{H}$. Model selection and Akaike's Information criterion (AIC): the general theory and its analytical extensions. Psychometrika. 1987;52:345-70.

39. Burns P. Robustness of the Ljung-Box test and its rank equivalent. SSRN 443560. 2002

40. Fuenzalida $H_{1}$ Rosenblüth B. Prewhitening of climatological time series. J Clim. 1990;3:382-93.

41. Alegana V, Wright J, Nahzat S, Butt W, Sediqi A, Habib N, et al. Modelling the incidence of Plasmodium vivax and Plasmodium falciparum malaria in Afghanistan 2006-2009. PLOS ONE. 2014:9:e102304.

42. Newbrander W, Ickx P, Feroz F, Stanekzai H. Afghanistan's basic package of health services: its development and effects on rebuilding the health system. Glob Public Health. 2014;9:S6-28. 
43. Ikram M, Powell C, Bano R, Quddus A, Shah S, Ogden E, et al. Communicable disease control in Afghanistan. Glob Public Health. 2013;9:543-57.

44. Howard N, Shafi A, Jones C, Rowland M. Malaria control under the Taliban regime: insecticide-treated net purchasing, coverage, and usage among men and women in eastern Afghanistan. Malar J. 2010;9:7.

45. Rowland M, Webster J, Saleh P, Chandramohan D, Freeman T, Pearcy B, et al. Prevention of malaria in Afghanistan through social marketing of insecticide-treated nets: evaluation of coverage and effectiveness by cross-sectional surveys and passive surveillance. Trop Med Int Health. 2002;7:813-22.

46. UNAMA. Afghanistan's health ministry reports significant decrease in malaria cases. https://unama.unmissions.org/afghanistan\%E2\%80\%99shealth-ministry-reports-significant-decrease-malaria-cases. Accessed 16 Oct 2016.

47. Reiner RC, Geary M, Atkinson PM, Smith DL, Gething PW. Seasonality of Plasmodium falciparum transmission: a systematic review. Malar J. 2015;14:343.
48. Palka EJ. Afghanistan: geographic perspectives. Dushkin Pub Group; 2004

49. Noden B, Kent M, Beier J. The impact of variations in temperature on early Plasmodium falciparum development in Anopheles stephensi. Parasitology. 1995;111:539.

50. Adimi F, Soebiyanto RP, Safi N, Kiang R. Towards malaria risk prediction in Afghanistan using remote sensing. Malar J. 2010;9:125.

51. Tolo News Agency. Rise in malaria a concern in Eastern Afghanistan. http://www.tolonews.com/en/afghanistan/25020-rise-in-malaria-aconcern-in-eastern-afghanistan. Accessed 25 Sept 2016.

52. Huang F, Zhou S, Zhang S, Wang H, Tang L. Temporal correlation analysis between malaria and meteorological factors in Motuo County Tibet. Malar J. 2011;10:54.

53. Tian L, Bi Y, Ho S, Liu W, Liang S, Goggins W, et al. One-year delayed effect of fog on malaria transmission: a time-series analysis in the rain forest area of Mengla County, south-west China. Malar J. 2008;7:110.

54. Mendelsohn R, Dinar A, Williams L. The distributional impact of climate change on rich and poor countries. Environ Dev Econ. 2006;11:159-78.

\section{Submit your next manuscript to BioMed Central and we will help you at every step:}

- We accept pre-submission inquiries

- Our selector tool helps you to find the most relevant journal

- We provide round the clock customer support

- Convenient online submission

- Thorough peer review

- Inclusion in PubMed and all major indexing services

- Maximum visibility for your research

Submit your manuscript at www.biomedcentral com/submit 